\title{
Development of Indomethacin Sustained Release Microcapsules using Ethyl Cellulose and Hydroxy Propyl Methyl Cellulose Phthalate by $0 / \mathrm{W}$ Emulsification
}

\author{
Md. Abu Hena Mostafa Kamal ${ }^{1}$, Maruf Ahmed ${ }^{1}$, Mir Imam Ibne Wahed ${ }^{1}$, \\ Md. Shah Amran ${ }^{2}$, Sharif Md. Shaheen ${ }^{1}$, Mamunur Rashid ${ }^{1}$ \\ and Md. Anwar-UI-Islam ${ }^{1}$ \\ ${ }^{1}$ Department of Pharmacy, University of Rajshahi, Rajshahi-6205, Bangladesh \\ ${ }^{2}$ Department of Pharmaceutical Chemistry, Faculty of Pharmacy, University of Dhaka, \\ Dhaka-1000, Bangladesh
}

\begin{abstract}
Indomethacin (IM) sustained release microcapsules were successfully prepared using ethyl cellulose (EC) and hydroxy propyl methyl cellulose phthalate (HPMCP) by o/w emulsification-solvent evaporation technique. The prepared microcapsules were evaluated for size, shape, drug content and in vitro drug release. The microcapsules show sustained release curves at $\mathrm{pH} 7.2$ phosphate buffer for up to $6 \mathrm{~h}$. The data obtained from the dissolution profiles were compared in the light of different kinetics models and the regression coefficients were compared. The in vitro dissolution study confirmed the Higuchi-order release pattern. Particle size and release data analysis from five consecutive batches prepared in the laboratory indicated suitable reproducibility of the solvent evaporation process. The release rate increased exponentially with the addition of HPMCP in EC. IM release rate was observed highest with the highest concentration of HPMCP (3:7 ratio of EC:HPMCP), used in the present studies. On the other hand, IM release rate was lowest when EC and HPMCP combination was used at the ratio of 10:0. When percent of HPMCP was increased, the particle size of microcapsules was decreased.
\end{abstract}

Key words: Indomethacin, sustained release, microcapsule, HPMCP, EC, Higuchi-order

\section{INTRODUCTION}

Sustain action dosage forms are designed to achieve a prolonged therapeutic action by continuously releasing medication over an extended period of time after administration of single dose. Microencapsulation is a process for coating of small solid particle; liquid droplets or dispersions using polymeric material to produce small particles of 1 to 5000 micrometer in size. It is a rapidly expanding technology for achieving sustained release dosage forms. Microencapsulation is used to modify and retard drug release. ${ }^{1}$ It involves the coating of the

Correspondence to: Maruf Ahmed

Tel: +88-0721-750041-49/4110

Fax: +88-0721-750064

Email: amaruf2003@yahoo.co.jp

Dhaka Univ. J. Pharm. Sci. 7(1): 83-88, 2008 (June) individual drug particles in inert polymeric material, through which the drug would diffuse at a controlled and predictable rate to the surrounding medium. Other techniques used colloidal polymer dispersions in a completely aqueous environment as an alternative to conventional microencapsulation techniques, which use organic solvents. ${ }^{2}$

Encapsulation and sustained release of indomethacin (IM), using $\mathrm{CO}_{2}$-based microencapsulation was studied by Liu and co-workers. ${ }^{3}$ The $\mathrm{CO}_{2}$ plasticizes the biodegradable polymers, increasing the drug diffusion rate in the particles so that drug loading is enhanced. Rowe and Carless studied the comparison of the in vitro dissolution behaviour of various indomethacin formulations with their in vivo 
bioavailability. The effects of the core to colloid wall ratio and particle size of the core on the in vitro release of indomethacin microcapsules prepared by the gelatin-acacia complex coacervation process have been examined. ${ }^{4}$ Dissolution behaviour of sustained release formulations of indomethacin with Eudragit RS was also studied by Khanfar and co-workers. ${ }^{5}$ In another study, complex coacervation of chitosan and carboxymethylcellulose was prepared to control the release of indomethacin from microcapsule. The mechanism lies in crosslinking between chitosan and carboxymethylcellulose with glutaraldehyde. ${ }^{6}$

Indomethacin, a nonsteroidal anti-inflammatory agent, has a short biological half-life of 2.6-11.2 hours. ${ }^{7}$ The usual oral dosage for adults is 25 or 50 mg, 2 to 3 times a day. Controlled release preparations of this drug are to increase patient compliance and to reduce adverse effects, fluctuation in plasma concentration and dosing frequency. Therefore, the objective of this study was to investigate the preparation of microcapsules using ethyl cellulose (EC) and hydroxy propyl methyl cellulose phthalate (HPMCP) by o/w emulsificationsolvent evaporation technique. The effects of EC and HPMCP ratio on the physical properties and drug release pattern of the pharmaceutical microcapsule were studied.

\section{MATERIALS AND METHODS}

Materials. Ethyl Cellulose (EC), Hydroxy Propyl Methyl Cellulose Phthalate (HPMCP) and Gelatin were purchased from Fluka, Switzerland. Indomethacin (product grade) was a kind gift from Novartis (BD) Ltd. The solvents and reagents were of analytical grade.

Preparation of indomethacin microcapsules. Microcapsules were prepared by an emulsification and organic solvent evaporation technique reported earlier by Jalil and Nixon. ${ }^{8}$ The apparatus used in this technique was very simple, consisting of stirrer, a flat bottomed glass vessel and thermostatically controlled water bath.
Five batches of microcapsules were prepared. In each batch amount of IM was $1 \mathrm{gm}$. and total amount of polymer was 2 gms. The details of the polymer proportion and their amount in different batches are shown in Table 1.

Table 1. Formulation of indomethacin microcapsules.

\begin{tabular}{|c|c|c|c|c|}
\hline \multirow[b]{2}{*}{ Batch } & \multirow[b]{2}{*}{$\begin{array}{l}\text { IM } \\
(\mathrm{g})\end{array}$} & \multicolumn{2}{|c|}{ Polymer } & \multirow{2}{*}{$\begin{array}{c}\text { Average } \\
\text { size } \\
(\mu \mathrm{m})\end{array}$} \\
\hline & & $\begin{array}{c}\text { Ratio (EC : } \\
\text { HPMCP) }\end{array}$ & $\begin{array}{c}\text { Amount } \\
(\mathrm{EC}+\mathrm{HPMCP}) \\
(\mathrm{g})\end{array}$ & \\
\hline 1 & 1 & $10: 0$ & $2.0+0.0$ & 87.7 \\
\hline 2 & 1 & $8: 2$ & $1.6+0.4$ & 65.5 \\
\hline 3 & 1 & $7: 3$ & $1.4+0.6$ & 40.0 \\
\hline 4 & 1 & $5: 5$ & $1.0+1.0$ & 22.9 \\
\hline 5 & 1 & $3: 7$ & $0.6+1.4$ & 18.6 \\
\hline
\end{tabular}

Each microcapsule batch weighs $3 \mathrm{gms}$. The polymer ratio and amounts in the microcapsules containing EC and HPMCP prepared by emulsification and organic solvent evaporation technique.

Continuous phase was gelatin gel in distilled water and dispersed non-aqueous phase consisted of polymers (EC and HPMCP) and IM solution in dichloromethane. At first $2 \% \mathrm{w} / \mathrm{v}$ gelatin solution in $500 \mathrm{ml}$ of distilled water was prepared. Then one gram IM and required amounts of polymers were dissolved in $20 \mathrm{ml}$ of dichloromethane. Two percent (2\%) gelatin solution was diluted with distilled water to obtain $0.5 \%$ gelatin solution.

A flat bottomed glass cylinder was partially immersed in a thermostatic water bath at $40^{\circ} \mathrm{C} .50 \mathrm{ml}$ of $0.5 \%$ gelatin solution was taken into that glass cylinder and kept for 10 minutes to equilibrate the temperature. The dispersed phase was then added and stirred with a stainless steel propeller at $2000 \mathrm{rpm}$. Agitation was continued for 45 minutes to ensure complete evaporation of the volatile solvent and formation of microcapsules. Finally microcapsules were separated by vacuum filtration. They were washed four times with $20 \mathrm{ml}$ of distilled water and dried in a desiccator.

Percentages of drug entrapment and drug recovery. The percentage of drug entrapment was calculated from the content of indomethacin in microcapsule determined according to the monograph of Indomethacin Extended Release Capsule in the 
USP XXIV. The percentage of drug recovery was computed from the following equation.

$\%$ Recovery $=M_{t} \cdot D_{m} 100 / M_{a} D_{i}$

where $D_{m}, M_{t}, D_{i}$ and $M_{a}$ were the drug content in microcapsule, the weight of total microcapsule yield and the initial amount of drug used in the microencapsulation process and weight of the determined microcapsule, respectively.

In vitro Dissolution Studies. Microencapsulated IM showed better drug release profile with USP paddle method than that of USP basket method. Simulated intestinal fluid ( $\mathrm{pH}$ 7.2) was an appropriate medium to use for dissolution studies on prolongedrelease microcapsules. ${ }^{9}$ In our study, the dissolution studies of approx. $200 \mathrm{mg}$ of microcapsules from each formulation were carried out using an "Electrolab Dissolution Tester USP (XXI) TDT-06". The paddle rotation was set at $50 \mathrm{rpm}$ and the temperature was controlled at $37 \pm 2{ }^{\circ} \mathrm{C}$ using 1 litre of dissolution medium of $\mathrm{pH} 7.2$ as chamber volume containing potassium dihydrogen phosphate, $\mathrm{KH}_{2} \mathrm{PO}_{4}$ $(0.2 \mathrm{M})$; sodium hydroxide, $\mathrm{NaOH}(0.2 \mathrm{M})$ and water. Time was recorded as soon as the microcapsules were put into the dissolution vessels. A five $\mathrm{ml}$ sample solution was withdrawn from each vessel at appropriate time intervals $(5,10,20,30,40,60,90$, $120,150,180,210,240,270,300,330,360$ minutes) for the analysis of drug release. Five $\mathrm{ml}$ of fresh phosphate buffer solution previously heated to $37^{\circ} \mathrm{C}$ was immediately added to the dissolution medium for compensating the sampling. The dissolution study was carried out for three samples from each formulation.

Size and size distribution. The size and size distribution were determined using light microscopy. The particle size was determined by measuring the Martin's diameter. ${ }^{10}$

Analysis of drug release. Drug released in the sample solution was determined spectrophotometrically by measuring the absorbance at $318 \mathrm{~nm}$ using a UV-visible spectrophotometer, the reference being the fresh dissolution medium of $\mathrm{pH}$ 7.2. The amounts of drug present in the samples were calculated with the help of appropriate calibration curves constructed from reference standards. Data analysis and statistical calculations were performed using a computer program GraphPad Prism version 3.00 for Windows (GraphPad Software, San Diego, CA, USA).

\section{RESULTS AND DISCUSSION}

Microcapsule size. IM microcapsules were prepared from EC and HPMCP by emulsificationsolvent evaporation technique in which different ratios of EC and HPMCP were used keeping the drug load constant. Microcapsules of same formulation were more or less uniform in size with spherical or oval shape when observed in a light microscope. The average sizes of IM microcapsules are given in Table 1. This observation clearly indicates that when percent of HPMCP was increased, the particle size of microcapsules was decreased. Figures 1a, 1b, 1c, 1d and 1e show the IM microcapsules of five batches respectively.

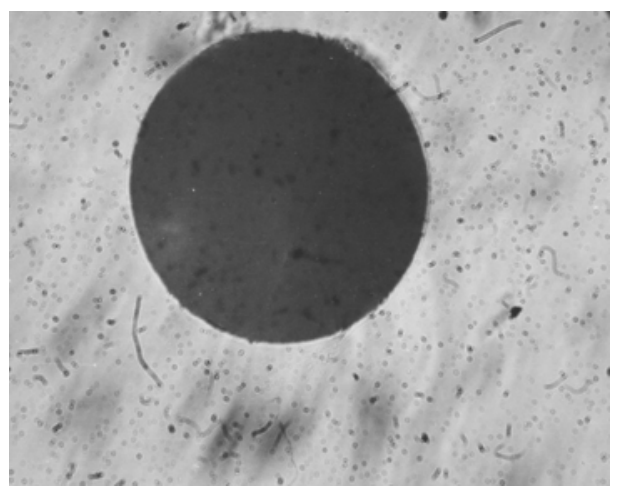

Figure 1(a), Microphotograph of EC and HPMCP combination microcapsules ratio $10: 0$

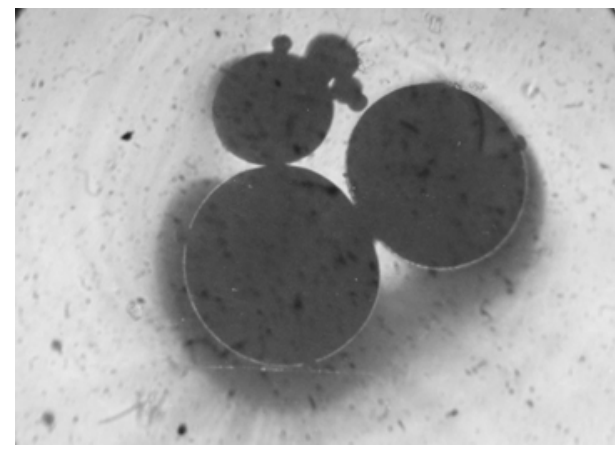

Figure 1(b), Microphotograph of EC and HPMCP combination microcapsules ratio $8: 2$ 


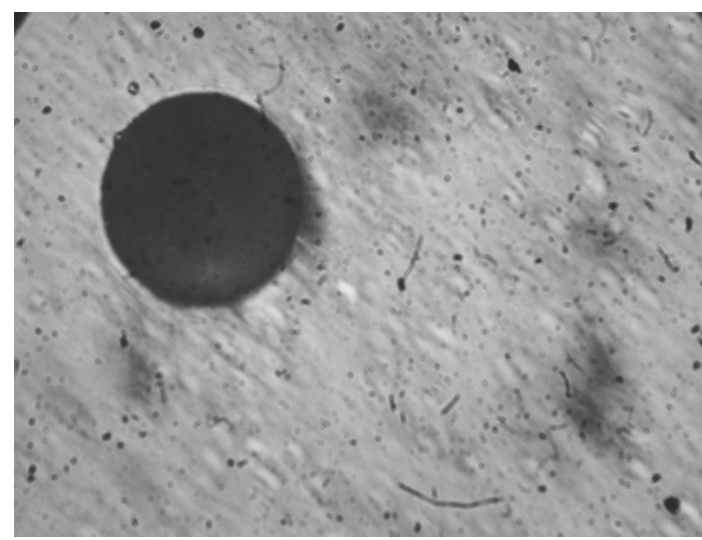

Figure 1(c), Microphotograph of EC and HPMCP combination microcapsules ratio $7: 3$

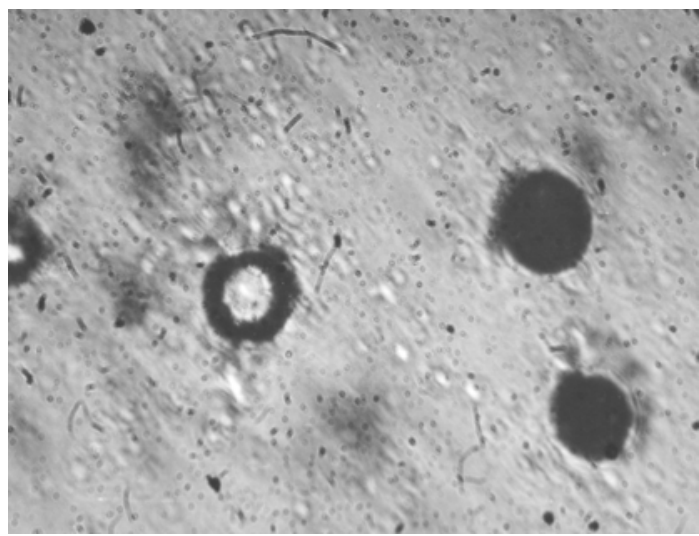

Figure 1(d), Microphotograph of EC and HPMCP combination microcapsules ratio $5: 5$

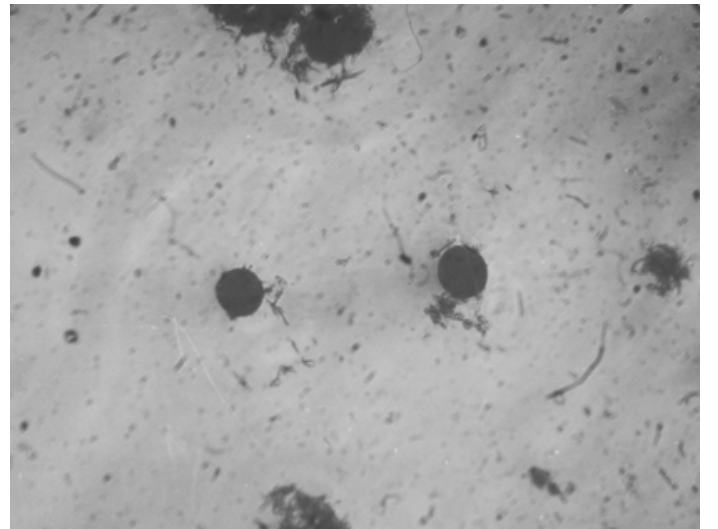

Figure 1(e), Microphotograph of EC and HPMCP combination microcapsules ratio $3: 7$

Drug entrapment and drug recovery. Drug entrapment in EC-HPMCP microcapsules was within a range of 66.17 to $89.67 \%$ as listed in Table 2. In contrast, the percentage of drug recovery shown in
Table 2 was obviously varied between 54.87 to $86.33 \%$. It could be seen that the drug recovery was influenced by the EC solution. It was also noted that high drug recovery could be achieved by increasing the concentration of EC solution. This might be due to increased thickness of EC coating of the microcapsules. However, the drug entrapment did not show any relationship with the concentration of EC, since it depends on the processing of microcapsule rather than on any ingredient of the formulation.

Table 2. Formulation variable affecting drug entrapment and drug recovery in indomethacin microcapsules

\begin{tabular}{cccc}
\hline Batch & $\begin{array}{c}\text { EC } \\
(\mathrm{gm})\end{array}$ & $\begin{array}{c}\text { \% Drug entrapment } \\
\pm \text { SEM }\end{array}$ & $\begin{array}{c}\text { \% Drug recovery } \\
\pm \text { SEM }\end{array}$ \\
\hline 1 & 2.0 & $89.67 \pm 0.55(3)$ & $86.33 \pm 0.72(3)$ \\
2 & 1.6 & $78.00 \pm 0.67(3)$ & $76.95 \pm 0.43(3)$ \\
3 & 1.4 & $84.45 \pm 1.02(3)$ & $77.23 \pm 0.65(3)$ \\
4 & 1.0 & $75.86 \pm 0.47(4)$ & $63.37 \pm 1.23(4)$ \\
5 & 0.6 & $66.17 \pm 0.18(4)$ & $54.87 \pm 0.88(4)$ \\
\hline
\end{tabular}

Numbers in parentheses indicate number of determinations

Drug release kinetics from EC-HPMCP microcapsules. IM release kinetics from microcapsules were studied using phosphate buffer $(\mathrm{pH} 7.2)$ at $37^{\circ} \mathrm{C}$ temperature and $50 \mathrm{rpm}$ paddle speed using a USP dissolution test apparatus. The release rate was slower when the content of EC was increased. A linear relation exists between the rate of release and the reciprocal amount of EC used for coating, indicating that the drug release was controlled by the coating thickness. When percent release was plotted against time (Figure 2), no straight lines were obtained indicating that IM release did not follow the zero order kinetics. First order plot ( $\log \%$ remaining vs. time) also showed curved released pattern (Figure 3).

In contrast, when percent release was plotted against square root of time (Figure 4), release profile showed linear relationship. The Higuchi square root equation describes the release from systems where the solid drug is dispersed in an insoluble matrix, and the rate of drug release is related to the rate of drug diffusion. ${ }^{11,12}$ This confirms that EC-HPMCP microcapsules were released in a Higuchian diffusion fashion. 


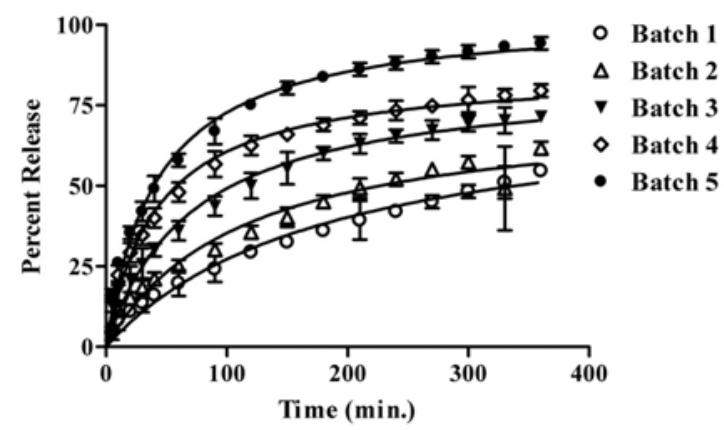

Figure 2. Zero order release profile of IM from polymeric microcapsules. Batch formulation is available in Table 1

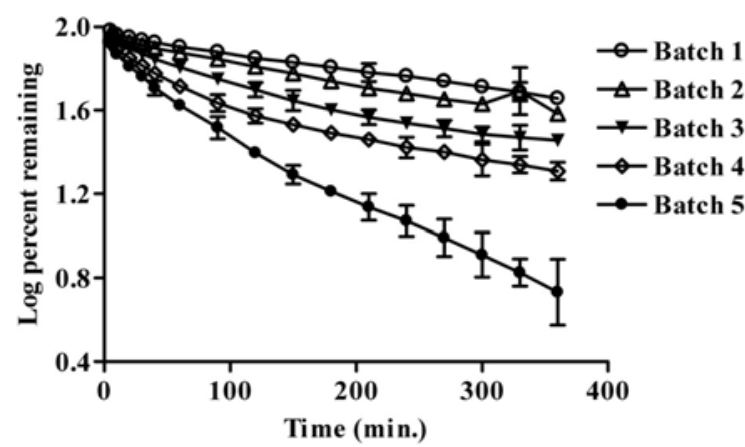

Figure 3. First order release profile of Ibuprofen from polymeric microcapsules. Batch formulation is available in Table 1

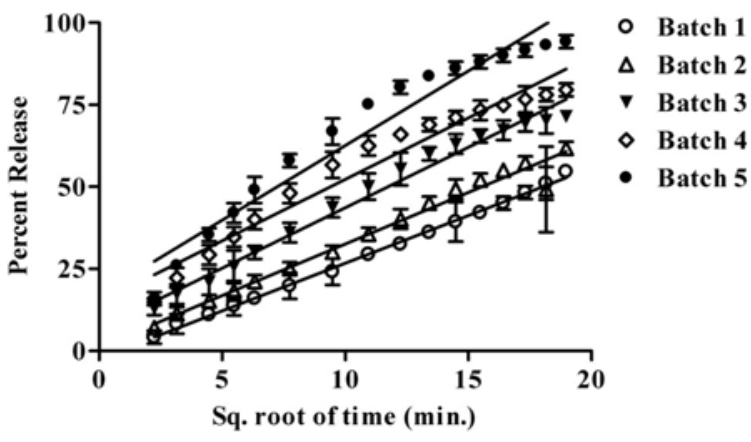

Figure 4. Higuchi release profile of IM from polymeric microcapsules. Batch formulation is available in Table 1

The slope values (Higuchi release rate constants, $\mathrm{K}_{\mathrm{h}}$ ) obtained from the straight line portion of the Figure 4 were $2.68,3.17,4.33,5.86$, and 6.92 for the formulation of $1,2,3,4$, and 5 respectively. These values were plotted against the percent of HPMCP content in the EC-HPMCP microcapsules and Figure 5 was obtained. This figure indicated that the release rate was increased with the increase of percent of HPMCP.

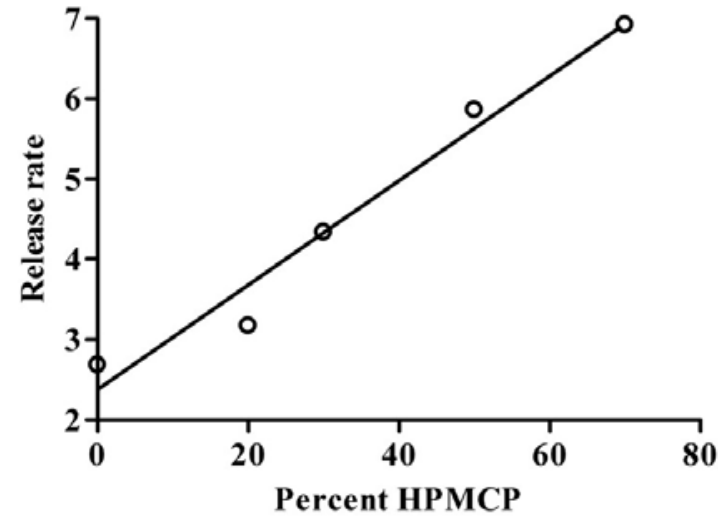

Figure 5. Release rate of IM from different proportion of HPMCP in microcapsules

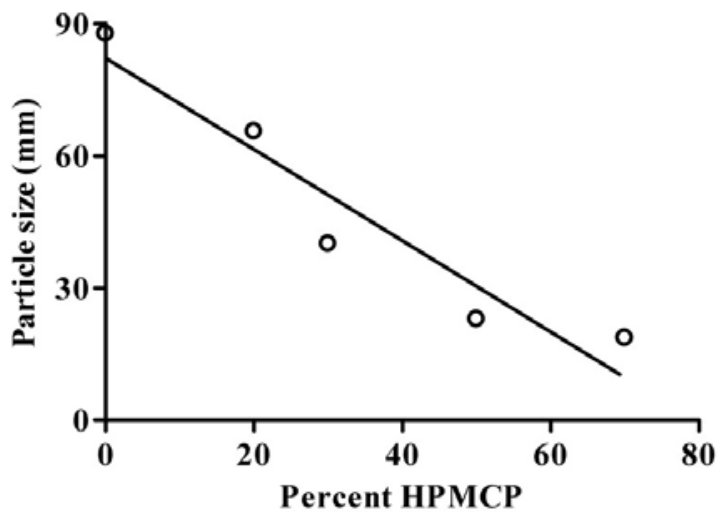

Figure 6. Particle size of IM microcapsules from different proportion of EC:HPMCP in microcapsules

The reason for gradually decreasing release rate may be due to the plasticization of the EC polymer thereby forming smooth and nonporous films around the core IM crystal. The increase in "Higuchian" release rate constant with the increase of HPMCP proportion to EC was due to higher rate of permeability of HPMCP in the dissolution medium of $\mathrm{pH} 7.2$ as well as increased surface area due to decreased particle size. Another study with the surface morphology of the microcapsules of IM, microencapsulated with EC, was examined using scanning electron microscopy, the microcapsules were found porous and spherical, and their porosity increased with increasing viscosity of EC. ${ }^{12}$ So, EC might have created porosity in its coating in this formulation also. The release rate of the drug from microcapsules decreased as the viscosity of EC decreased. The release rate also decreased with increasing microcapsule size. 
The calculated correlation coefficient $\left(\mathrm{R}^{2}\right)$ of the percent drug release versus square root of time was between 0.9417-0.9984 whereas those of the percent drug release versus time and log percent drug remained versus time were between 0.8206-0.9748 and $0.8917-0.9584$, respectively. This implied that the release of drug from the prepared microcapsules accordingly followed Higuchi model.

The particle size of the microcapsules also decreased with increasing HPMCP content and decreasing EC content (Figure 6) indicating that with the decrease of EC, the coating thickness is also decreased resulting in decreased size of microcapsules.

\section{CONCLUSIONS}

The hydrophobic drug, indomethacin, has been successfully incorporated into microcapsules of EC and HPMCP by o/w emulsification-solvent evaporation technique. Microcapsules of same batch have similar sizes and shapes when observed in a light microscope. In vitro dissolution study shows that the drug release followed Higuchi-order or diffusion controlled manner. This was statistically proved from the release curves by comparing their correlation coefficients. A linear relationship exists between the rate of release and the reciprocal amount of ethyl cellulose used for coating, indicating that the drug release was controlled by the coating thickness. The percent drug recovery was also found to be high and increased with the concentration of EC. The EC:HPMCP microcapsules were thus found suitable for controlled and sustained release products in vitro. However, a formulation of indomethacin cannot be considered as sustained release entity unless the in vivo study demonstrates a concentration/response relationship together with a short plasma elimination half-life and inactive or uncharacterized activity of any generated metabolites and produce transient side effects associated with dissolution and/or peak concentrations. ${ }^{13}$ Therefore, an extensive in vivo study is essential to make this sustained-release microcapsule formulation of IM to be used clinically.

\section{REFERENCES}

1. Bakan, J.L. 1986. In: The Theory and Practice of Industrial Pharmacy. (Lachman, L., Lieberman, H.A. and Kanig, J.L., Eds.), Lea and Febiger, Philadelphia, Chapter 13-part III, pp. 412-429.

2. Bodmeier, R. and Wang, J. 1993. Microencapsulation of Drugs with Aqueous Colloidal Polymer Dispersions. J. Pharm. Sci. 82, 191-94.

3. Liu, H., Finn, N., Yates, M.Z. 2005. Encapsulation and Sustained Release of a Model Drug, Indomethacin, Using $\mathrm{CO}_{2}$-Based Microencapsulation. Langmuir 21, 379-385.

4. Rowe, J.S. and Carless, J.E. 1981. Comparison of the In Vitro Dissolution Behaviour of Various Indomethacin Formulations with Their In vivo Bioavailability. J. Pharm. Pharmacol. 33, 561-564.

5. Khanfar, M.S., Salem, M.S., Najib, N.M. and Pillai, G.K. 1997. Dissolution Behaviour of Sustained Release Formulations of Indomethacin with Eudragit RS. Acta. Pharm. Hung. 67(6), 235-239.

6. Tiyaboonchai, W. and Ritthidej, G.C. 2003. Development of Indomethacin Sustained Release Microcapsules Using Chitosan-Carboxymethylcellulose Complex Coacervation. Songklanakarin J. Sci. Technol. 25, 245-254.

7. Flower, R.J., Moncada, S. and Vane, J.R. 1990. Indomethacin. In: Goodman and Gilman's The Pharmacological Basis of Therapeutics (Gilman, A.G. et al. Eds.), Macmillan Publishing Co., New York, pp. 695-97.

8. Jalil, R. and Nixon, J.R. 1990. Microencapsulation Using Poly (L-Lactic Acid) II: Preparative Variables Affecting Microcapsule Properties. J. Microencapsul. 7, 25-39.

9. Zour, E. and Lausier, J.M. 1984. Dissolution Studies of Microencapsulated Indomethacin; Effect of Method and Medium on Dissolution. J. Microencapsul. 1, 47-51.

10. Martin, A. 1993. Colloids. In: Physical Pharmacy: Physical and Chemical Principles in Pharmaceutical Sciences. 4th ed., Lea and Febiger, Philadelphia, pp. 393-422, 453-476.

11. Baveja, S.K., Ranga Rao K.V. and Padmalatha Devi K. 1987. Zero-order release hydrophilic matrix tablets of $\beta$-adrenergic blockers. Int. J. Pharm. 39, 39-45.

11. Schwartz, J.B., Simonelli, A.P. and Higuchi, W.I. 1968. Drug release from wax matrices. I. Analysis of data with first-order kinetics and with the diffusion-controlled model. J. Pharm. Sci. 57, 274-277.

12. Lee, H.J., Lee, M.H. and Shim, C.K. 1984. Preparation and Evaluation of Ethyl Cellulose Microcapsules of Indomethacin. Arch. Pharm. Res. 7, 33-40.

13. Green, J.A. 1984. Indomethacin sustained-release? Drug Intell. Clin. Pharm.18, 1004-1007. 